\title{
Feasibility of an Interdisciplinary Early Intervention for Patients with Low Levels of Responsiveness Following an Acquired Brain Injury
}

\author{
Treena Seeto, ${ }^{1}$ Suzanne Kuys, ${ }^{2,3}$ Cassandra Budden, ${ }^{1}$ Erin Griffin, ${ }^{1,4}$ Haylee Kajewski, ${ }^{1,4}$ \\ and Steven McPhail ${ }^{5,6}$ \\ 1 Princess Alexandra Hospital, Brisbane, Australia \\ 2 The Prince Charles Hospital, Brisbane, Australia \\ ${ }^{3}$ Griffith Health Institute, Griffith University, Gold Coast, Australia \\ 4 Jacana Acquired Brain Injury Service, Brisbane, Australia \\ ${ }^{5}$ Centre for Functioning and Health Research, Metro South Health, Brisbane, Australia \\ ${ }^{6}$ Institute of Health and Biomedical Innovation and School of Public Health, Queensland University of \\ Technology, Brisbane, Australia
}

\begin{abstract}
There is a scarcity of research investigating interdisciplinary early rehabilitation intervention models for people with low levels of responsiveness following acquired brain injury. This is particularly problematic for service models in acute hospital settings where early rehabilitation of such patients may be assigned a low priority in comparison to other competing demands. This pilot study investigated the feasibility of implementing an interdisciplinary early rehabilitation intervention in an acute neurosciences ward within existing staffing, and describes clinical changes among intervention participants. A prospective longitudinal study, with a matched historical control (individually matched by Glasgow Coma Scale) was undertaken. Prospective intervention participants $(n=13)$ received up to twiceweekly interdisciplinary intervention in addition to usual care. Matched historical controls $(n=13)$ received only usual care. Implementing an interdisciplinary early intervention proved feasible in this acute care setting. Significant improvement (alpha $=.05$ ) among the patients receiving the interdisciplinary intervention was observed across all clinical outcomes, including: Coma Recovery Scale-Revised, Glasgow Coma Scale, Mental Status Questionnaire, Royal Brisbane Hospital Outcome Measure for Swallowing, selected subtests of the Functional Independence Measure, Acquired Brain Injury Physiotherapy Assessment and the Clinical Outcomes Variable Scale. A future randomised clinical trial is warranted.
\end{abstract}

Keywords: interdisciplinary, low levels of responsiveness, brain injury, rehabilitation, impaired consciousness

\section{Introduction}

Following severe brain injury, rehabilitation for those people with minimal or low levels of responsiveness is still recommended, although expected recovery is anticipated to be slow (Giacino et al., 2002; National Health and Medical Research
Council, 2008). Rehabilitation provision should be multidisciplinary, be commenced as early as possible and include therapeutic interventions which focus on repeated and controlled exposure to sensory stimuli (physical, oral, visual, auditory and olfactory), with a view to increasing a person's 
volitional response (Meyer et al., 2010; TurnerStokes, Disler, Nair, \& Wade, 2005), prevent secondary problems and educate families (Das-Gupta $\&$ Turner-Stokes, 2002; National Health and Medical Research Council, 2008).

Consideration of rehabilitation is particularly relevant for patients with low levels of responsiveness who may be overlooked in traditional models of rehabilitation delivery due to a perceived inability to participate (Katz, Polyak, Coughlan, Nichols, $\&$ Roche, 2009). Neuroplasticity benefits and subsequent functional outcomes can be optimised with early rehabilitation (Lippert-Gruner, Wedekind, \& Klug, 2002; Reid-Arndt, Schopp, Brenneke, Johnstone, \& Poole, 2007). In fact, following severe brain injury, patients who receive early intensive rehabilitation experience shorter acute and rehabilitation lengths of stay, fewer residual impairments and have better function on discharge from hospital rehabilitation and at 2-year follow-up (TurnerStokes, 2008; Turner-Stokes et al., 2005), as well as an improved rate of discharge to home accommodation in the community (Mackay, Bernstein, Chapman, Morgan, \& Milazzo, 1992).

In contemporary hospital settings there may be a delay in the commencement of rehabilitation for people with low levels of responsiveness (Katz et al., 2009). The delay may be due to a perception that patients must possess a specific level of responsiveness to benefit from early rehabilitation. Additionally, limited bed availability in a rehabilitation unit may result in some patients remaining on an acute hospital ward with limited therapy while waiting for transfer to a specialist brain injury rehabilitation unit (Shiel et al., 2001). This may be particularly problematic for patients with low levels of responsiveness in an acute care setting who could be assigned a lower priority for rehabilitation than patients with higher levels of consciousness.

Potential benefits of implementing early rehabilitation in acute hospital ward settings prior to patients' transfer to a rehabilitation ward appear evident. However, limited therapy resources and a high level of competing demands in acute care settings may mean that this undertaking is complex. One potential solution worthy of investigation is the utilisation of interdisciplinary therapies delivered in a combined context; that is, providing treatment for a single patient by therapists working together simultaneously with planned goals and treatment plan. Such an approach has the potential to provide a coordinated therapeutic intervention, improve the efficiency of clinical staff while providing early rehabilitation to patients with low levels of responsiveness in acute hospital settings.
The aims of this study were to:

- examine the feasibility of implementing an interdisciplinary early intervention for people with low levels of responsiveness in an acute care setting (where feasibility refers to the ability to implement the intervention within existing clinical human resources);

- describe changes in important clinical outcomes among patients receiving this intervention; and

- examine whether duration of hospital length of stay and hospital transfer pathway was comparable to matched historical controls.

\section{Method}

\section{Design}

A prospective longitudinal study with matched historical controls was implemented with pre-post intervention assessment of clinical outcomes by an assessor independent of the treating clinicians, but not blinded to the prospective intervention delivery.

\section{Participants and Setting}

Participants included patients following severe brain injury with low levels of responsiveness admitted to the Neurosciences Unit at the Princess Alexandra Hospital, Brisbane from March 2011 to March 2012. This unit incorporates eight highdependency beds and an additional 28 acute beds. Allied health staffing for this unit includes two fulltime physiotherapists, occupational therapists and social workers, one full-time speech pathologist, a 0.5 full-time-equivalent dietitian and a 0.7 fulltime-equivalent physiotherapy assistant. For inclusion in the interdisciplinary intervention, patients with low levels of responsiveness following a severe brain injury needed to be medically stable, require more than one allied health discipline intervention, have an estimated length of hospital stay of greater than 4 weeks, and were considered by the treating clinical team to be likely to receive a referral for a rehabilitation suitability assessment. Patients were excluded if they were deemed to be palliative or for immediate repatriation to another hospital.

Historical control participants were identified from patients discharged from this neurosciences unit within the previous 36 months, matched by neurosciences unit admission Glasgow Coma Scale (GCS; Teasdale \& Jennett, 1974) score (within 2 points) to minimise differences in neurological function on admission to the unit between the two groups (Gill, Reiley \& Green, 2004; 
Husson, Ribbers, Willemse-van Son, Verhagen, \& Stam, 2010). The full-time-equivalent clinical staffing and patient bed numbers for the unit remained constant throughout the duration of the study.

\section{Intervention}

Prospectively recruited participants received the interdisciplinary intervention. This interdisciplinary intervention was aimed at a new service model where the treating physiotherapist, occupational therapist and speech pathologist worked together with the patient and their family to provide a targeted therapy programme addressing individualised physical and respiratory needs, swallowing and communication disorders, provide structured sensory stimulation, and functional task retraining. Twice-weekly 60-90 minute interdisciplinary intervention sessions in addition to usual care were conducted, typically in the physiotherapy gym. Treatment plans were individualised based on goals identified by the patient's relevant family members and treating therapists, and included activities such as structured sensory stimulation focusing on increasing alertness and attention or following commands more consistently; functional task retraining such as wiping the mouth, using a face towel, bringing cup/food to the mouth, or catching/tapping/throwing a ball/balloon while sitting up on the edge of a bed; passive mobilisation or sensory stimulation of limbs; tasting different tastes and/or consistencies of food/drink while facilitating more correct alignment in a supported sitting or standing position; transfer or early gait training; and speech 'drills'. Treating therapists were not blinded to the intervention received or participants' inclusion to the study. They were aware that clinical outcome measures were being recorded but were unaware of frequency and intensity of treatment measures being collated. Usual care, received by both participant groups, comprised a more traditional model of service delivery where disciplines delivered their intervention in isolation and at a frequency determined by each treating therapist as part of their routine clinical practice. Similar discipline-specific therapies were used in both groups.

\section{Baseline Demographic and Clinical Outcome Measures}

Basic clinical and demographic information was collected, including age at the time of injury, gender, injury type (traumatic versus non-traumatic), Glasgow Coma Scale (Prasad, 1996; Teasdale \& Jennett, 1974) score, Mental Status Questionnaire (MSQ; Wilson \& Brass, 1973), Royal Brisbane
Hospital Outcome Measure for Swallowing (Ward \& Conroy, 1999) score, whether a tracheostomy was in situ and number of days that the tracheostomy remained in situ was recorded. All measures were recorded on admission to the neurosciences unit.

Additionally, for the prospective intervention group a suite of clinical outcome measures was administered by an independent assessor, not involved in the delivery of the intervention, on recruitment to the study and immediately prior to discharge or transfer from the unit. The clinical outcome measures included Coma Recovery Scale-Revised (Kalmar \& Giacino, 2005), Glasgow Coma Scale (Teasdale \& Jennett, 1974), Mental Status Questionnaire (Wilson \& Brass, 1973), Functional Independence Measure subtests of eating, expression, comprehension, upper body dressing and transfers (bed, chair, wheelchair) (Kidd et al., 1995) to evaluate selected aspects of activities of daily living; Royal Brisbane Hospital Outcome Measure for Swallowing (Ward \& Conroy, 1999), Acquired Brain Injury Physiotherapy Assessment (Gesch, Nascimento, Passier, \& Haines, 2005) and the Clinical Outcomes Variable Scale (Low Choy, Kuys, Richards, \& Isles, 2002; Seaby \& Torrance, 1989).

To describe the patients' clinical pathway, the intensive care length of stay prior to neurosciences unit admission, the neurosciences unit length of stay and discharge or transfer destination were collected from hospital records. Feasibility of the implementation of the interdisciplinary intervention within an acute neurosciences unit was determined by the number of interdisciplinary sessions conducted for each patient, number of sessions attended by a family member, and the number of treatment sessions completed. The number of individual treatment sessions were manually collated from hospital records and confirmed by the hospital's electronic record of treatment sessions delivered by therapists working in the unit (where details of each therapy session are routinely recorded) for both the prospective and historical groups.

\section{Procedure}

Patients meeting the inclusion criteria were identified by the clinical staff of the neurosciences unit. For patients taking part in the interdisciplinary intervention, assessments including the suite of clinical outcome measures were conducted by the independent assessor prior to their participation in the group and again at discharge from the unit. To address the secondary aim, baseline clinical and demographic information, the number of treatment sessions received and the patients' clinical pathway 


\section{TABLE 1}

Demographic and Baseline Clinical Information for Participants

\begin{tabular}{|c|c|c|}
\hline & $\begin{array}{l}\text { Intervention group } \\
(n=13)\end{array}$ & $\begin{array}{l}\text { Historical group } \\
(n=13)\end{array}$ \\
\hline Age in years, median $\left(\mid Q^{a}\right)$ & $42(22-56)$ & $33(20-51)$ \\
\hline Males, number (\%) & $12(92 \%)$ & $8(62 \%)$ \\
\hline Traumatic injury, number (\%) & $9(69 \%)$ & $8(62 \%)$ \\
\hline $\begin{array}{l}\text { Glasgow Coma Scale at admission to the neurosciences unit, } \\
\text { median (IQR) }\end{array}$ & $9(6-10)$ & $7(5-10)$ \\
\hline $\begin{array}{l}\text { Neurosciences unit Admission Mental Status Questionnaire, /10, } \\
\text { median (IQR) }\end{array}$ & $0(0-0)$ & $0(0-0)$ \\
\hline $\begin{array}{l}\text { Time to neurosciences unit admission from injury, days, median } \\
\text { (IQR) }\end{array}$ & $18(12-22)$ & $12(8-18)$ \\
\hline $\begin{array}{l}\text { Neurosciences Admission Royal Brisbane Hospital Outcome } \\
\text { Measure for Swallowing, /10, median (IQR) }\end{array}$ & $1(1-1)$ & $1(1-1)$ \\
\hline $\begin{array}{l}\text { Patients with a tracheostomy in situ on admission to neurosciences } \\
\text { ward, number (\%) }\end{array}$ & $11(85 \%)$ & $12(92 \%)$ \\
\hline Days with tracheostomy in situ, median $(I Q R)^{b}$ & $40(34-60)$ & $28(25-46)$ \\
\hline
\end{tabular}

a Interquartile range.

bOnly includes patients admitted with tracheostomy.

details were collated for both the prospective and historical groups. Ethical approval for the study was obtained from the Metro South Human Research Ethics Committee. Informed consent was obtained from the next of kin prior to patient inclusion in the study.

\section{Data Analysis}

Conventional descriptive statistics (including median and interquartile range (IQR), as well as number and percentage (\%) where relevant) for baseline clinical information and demographic data were tabulated for patients in the prospective and historical groups (Table 1). Clinical outcome measures for the prospective group who received the interdisciplinary intervention were tabulated for their admission and discharge assessments (Table 2). Non-parametric statistical analyses were conducted due to an inability to confirm normality of distribution of data in the small datasets involved. Mann-Whitney $U$-tests were undertaken to examine the change in the clinical outcome measures between admission and discharge assessments for the prospective group. Conventional descriptive statistics were also used to describe the clinical pathway of patients in the intervention and historical control groups (Table 3). The median (IQR) number of interdisciplinary sessions received by the intervention group was calculated. The number (cumulative total) and frequency (sessions per week) of individual treatment sessions were tabulated for both groups (Table 4).

\section{Results}

A total of 14 participants were identified for recruitment to the prospective group. One patient was unexpectedly transferred to another acute unit shortly after admission and did not participate in the intervention or clinical assessments. This patient was excluded from the study. The remaining 13 participants completed all assessments, participated in the interdisciplinary intervention group and were included in analyses. An adverse event log was kept for any adverse events that occurred during (or potentially as a result of) participation in the interdisciplinary group intervention; however, no such events occurred. Thirteen matched historical control patients had routine baseline assessments and clinical information recorded in their hospital record and were included in analyses.

The clinical and demographic information for the prospective and control groups is presented in Table 1. Overall, both participant groups were comparable on admission to the neurosciences unit. Intervention group participants were older (median 42 versus median 33 years) and had more males in the group $(n=12)$ compared to the controls $(n=8)$. Non-traumatic causes of brain injuries across the two groups were varied and included subdural haematoma, subarachnoid haemorrhage, hypertensive intracranial haemorrhage, and a non-functioning infected shunt.

Clinical outcome measures for the initial and discharge assessment are presented in Table 2 for patients who received the interdisciplinary intervention. Outcome measure scores from the initial 
TABLE 2

Clinical Outcome Measures for the Early Intervention Group at Initial and Discharge Assessments

\begin{tabular}{|c|c|c|c|}
\hline Outcome measure (maximum score) & $\begin{array}{l}\text { Initial assessment } \\
\text { median }\left(I Q R^{a}\right)\end{array}$ & $\begin{array}{l}\text { Discharge } \\
\text { assessment } \\
\text { median (IQR) }\end{array}$ & $p$ value $^{b}$ \\
\hline Coma Recovery Scale (/23) & $18(8-23)$ & $23(18-23)$ & .02 \\
\hline Glasgow Coma Scale (/15) & $11(10-14)$ & $14(12-14)$ & .01 \\
\hline Mental Status Questionnaire (/10) & $0(0-3)$ & $5(0-9)$ & .02 \\
\hline $\begin{array}{l}\text { Royal Brisbane Hospital Outcome Measure for } \\
\text { Swallowing }(/ 10)\end{array}$ & $3(3-4)$ & $6(4-9)$ & .00 \\
\hline FIM eating (/7) & $1(1-1)$ & $5(1-6)$ & .02 \\
\hline FIM expression (/7) & $1(1-2)$ & $2(1-5)$ & .03 \\
\hline FIM comprehension (/7) & $2(1-3)$ & $3(1-5)$ & .01 \\
\hline FIM upper body dressing (/7) & $1(1-1)$ & $3(1-4)$ & .01 \\
\hline FIM transfers (/7) & $1(1-1)$ & $1(1-4)$ & .04 \\
\hline Acquired Brain Injury Physiotherapy Assessment (/60) & $40(34-41)$ & $46(36-51)$ & .01 \\
\hline Clinical Outcomes Variable Scale (/91) & $20(15-27)$ & $32(23-43)$ & .00 \\
\hline
\end{tabular}

a Interquartile range.

${ }^{b}$ Results of Mann-Whitney test.

FIM, Functional Independence Measure.

assessment indicate that this patient group experienced severe impairment across the range of included assessments. At discharge, patients continued to demonstrate substantial functional impairments; however, results of the Mann-Whitney $U$ tests indicated that each of the clinical outcomes significantly improved for all measures between the (unit) admission and discharge assessments ( $p$ values ranged from <.001 to .04).

The patient flow during hospital admission for patients in the prospective and historical groups is presented in Table 3. The severe nature and chronicity of the acquired brain injuries among both the prospective and historical groups is illustrated by the interquartile length of stay range for intensive care admission prior to being transferred to the neurosciences unit. This ranged from approximately 1 to 3 weeks across both groups. Despite a comparable median, the upper and lower quartiles for length of stay in the neurosciences unit were lower for the prospective intervention group (28-64 days) than the matched historical control group (44-174 days). Ten (77\%) of the prospective group patients were transferred to a brain injury specific rehabilitation unit, in comparison to 8 $(62 \%)$ of the historical control group.

TABLE 3

Patient Flow During Hospital Admission, Including Discharge or Transfer Destination Type

\begin{tabular}{lcc}
\hline & $\begin{array}{c}\text { Intervention group } \\
(n=13)\end{array}$ & $\begin{array}{c}\text { Historical } \\
\text { group }(n=13)\end{array}$ \\
\hline $\begin{array}{l}\text { Intensive care unit length of stay in days (prior to neurosciences } \\
\left.\text { unit admission), median (IQR }{ }^{a}\right)\end{array}$ & $18(12-21)$ & $11(8-17)$ \\
$\begin{array}{l}\text { Neurosciences unit length of stay in days, median (IQR) } \\
\text { Discharge destination: }\end{array}$ & $55(28-64)$ & $56(44-174)$ \\
$\begin{array}{l}\text { Brain injury rehabilitation unit, } n(\%) \\
\text { Brain injury specific slow to recover rehabilitation unit, } n(\%)\end{array}$ & $7(54 \%)$ & $7(54 \%)$ \\
$\begin{array}{l}\text { Non-brain injury specific rehabilitation unit, } n(\%) \\
\text { Home, } n(\%)\end{array}$ & $3(23 \%)$ & $1(8 \%)$ \\
Other acute hospital, $n(\%)$ & $1(8 \%)$ & $0(31 \%)$ \\
Interim care, $n(\%)$ & $1(8 \%)$ & $0(0 \%)$ \\
\hline
\end{tabular}

${ }^{a}$ Interquartile range. 


\section{TABLE 4}

Cumulative Total and Frequency (Sessions per Week) of Therapist-delivered Individual Treatment Sessions in Addition to Therapy Received as Part of the Group Intervention

\begin{tabular}{|c|c|c|c|}
\hline & $\begin{array}{l}\text { One-to-one treatment sessions received from } \\
\text { therapists during neurosciences unit admission }\end{array}$ & $\begin{array}{l}\text { Total number of sessions, } \\
\text { median }\left(I Q R^{a}\right)\end{array}$ & $\begin{array}{l}\text { Frequency per } \\
\text { week, median (IQR) }\end{array}$ \\
\hline \multirow{4}{*}{ 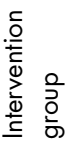 } & Total individual sessions delivered by therapists & $95(82-117)$ & $12.5(10.6-13.0)$ \\
\hline & Occupational therapy individual sessions & $20(13-27)$ & $2.8(1.6-3.5)$ \\
\hline & Physiotherapy individual sessions & $56(31-68)$ & $7.0(6.4-7.6)$ \\
\hline & Speech Pathology individual sessions & $27(16-38)$ & $2.4(2.0-3.2)$ \\
\hline \multirow{4}{*}{ 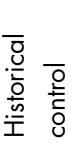 } & Total individual sessions delivered by therapists & $95(53-258)$ & $9.8(8.6-12.0)$ \\
\hline & Occupational therapy individual sessions & $16(9-31)$ & $1.8(1.1-2.3)$ \\
\hline & Physiotherapy individual sessions & $61(34-123)$ & $5.9(4.6-6.4)$ \\
\hline & Speech Pathology individual sessions & $23(14-71)$ & $2.6(2.2-3.5)$ \\
\hline
\end{tabular}

a Interquartile range.

Interdisciplinary early intervention participants received a median (IQR) of 5 (4-6) group treatment sessions. The median (IQR) number of group sessions attended by family members was 2 (1-5). The number and frequency of individual treatment sessions received from therapists as a component of usual care are presented in Table 4. The median total number of individual treatment sessions received was comparable for both groups. However, the IQR of total sessions received was larger for the historical control group (53-258) than the prospective intervention group (82-117); congruent with the shorter length of stay range observed among the prospective group (Table 3). The median, lower quartile and upper quartile frequency (individual sessions per week) were higher for the prospective group participants 12.5 (10.613.0) than the historical controls 9.8 (8.6-12.0).

\section{Discussion}

This investigation has been the first to demonstrate that implementing an interdisciplinary early intervention provided by physiotherapy, occupational therapy and speech pathology for people with low levels of responsiveness in an acute care setting can be feasibly implemented within the existing clinical staffing for the unit. Patients with low levels of responsiveness, their family members and allied health staff from multiple disciplines successfully participated in the intervention without any adverse events occurring. The observation of improvement in each of the clinical outcomes among the interdisciplinary intervention participants was positive (Table 2); although this cannot be attributed directly to the interdisciplinary intervention without accounting for natural recovery and other usual care interventions. The lower length of stay IQR in the neurosciences unit for the prospective intervention group (Table 3 ) and their slightly higher proportion of acceptance to brain injury specific rehabilitation (Table 3) were also encouraging. Although, this too should similarly only be interpreted with caveats in the context of the small sample size and non-randomised nature of the matched comparator group. Nonetheless, this investigation has successfully achieved its aims and generated novel empirical data worthy of further consideration.

The observed improvement in clinical outcomes among the group who received the interdisciplinary early intervention was diverse, substantial and clinically significant. This included improvement in arousal, level of consciousness and basic cognitive function (Table 2). For example, at the commencement of the interdisciplinary intervention, participants were not oriented to time, place or self (MSQ $=0 / 10), 50 \%$ were coping with their secretions but remained nil by mouth or had just commenced oral intake, and 50\% required total assistance to eat and to transfer. By discharge from the neuroscience unit, participants were partially oriented, mostly to self and place (MSQ $=5 / 10$ ), and $50 \%$ of participants had at least commenced oral intake (with some demonstrating swallowing function at pre-morbid level), were able to eat with modified independence, and provided up to $75 \%$ of effort required to complete transfers. The amount of positive change observed across these outcomes highlights the potential for patients with low levels of responsiveness to be able to experience improved orientation to time, place and self, and the ability to demonstrate memory and general knowledge. Similarly, the positive changes in swallowing, communication and motor performance outcomes highlight the potential for patients with 
impaired cognition to make meaningful functional gains (Table 2). Early interdisciplinary rehabilitation is likely to maximise these early functional gains and build a firm foundation for further rehabilitation interventions delivered in subacute brain injury specific rehabilitation settings.

Perhaps the most interesting unexpected finding was the higher frequency with which individual therapy sessions were delivered to patients participating in the interdisciplinary intervention group. The investigators held no favoured hypotheses regarding whether (or how) commencement of the group programme by the usual clinical staff would affect the delivery of individual treatment sessions for the patients with low levels of responsiveness outside of the intervention programme. It is plausible that the extra time demand created by planning and running the group would result in fewer opportunities for individual treatment sessions for patients participating in the intervention. In contrast, it is plausible that the increased focus on patients with low levels of responsiveness generated by the intervention could result in a higher priority being assigned to these patients. The higher frequency with which patients in the intervention group received individual treatment sessions outside of the group programme (Table 4) indicated that the second of these possible influences was the most likely to have occurred. Clinical staff may have intentionally (or unintentionally) assigned a higher priority to these patients with low levels of responsiveness, after seeing them in the interdisciplinary intervention setting; or in an effort to prepare them for participation in the interdisciplinary intervention. Another potential explanation is that the increased frequency of individual treatment sessions occurred as a result of a Hawthorne Effect (Adair, 1984; Mayo, 1933). Regardless of the causality of the increased early rehabilitation received by this group, the positive clinical outcomes (Table 2) and patient clinical pathway (Table 3) findings from this investigation add weight to the mounting evidence for early rehabilitation interventions for people who have suffered severe brain injury and have low levels of responsiveness.

Comparison with prior research is difficult, given the novel nature of providing an interdisciplinary (physiotherapy, occupational therapy and speech pathology) combined intervention to patients with low levels of responsiveness in an acute care hospital setting. However, previous research has demonstrated improvements in consciousness and functional tasks among patients with low levels of responsiveness who were provided with traditional therapies, pharmaceuticals, median nerve stimulation and neutraceuticals over a period of 12 weeks (DeFina et al., 2010). Similarly, the ben- efits observed in clinical outcomes among patients following the intervention were also consistent with other investigations among patients receiving acute and subacute rehabilitation therapies following severe acquired brain injuries (Cowen et al., 1995; Eilander et al., 2007; Katz et al., 2009; Sorbo et al., 2005).

Patients with low levels of responsiveness following severe brain injuries may be considered inappropriate candidates for rehabilitation (Katz et al., 2009). This may occur due to an uncertain or poor prognosis and a potentially unfounded assumption they will not experience benefits from rehabilitation (Katz et al., 2009). In addition to demonstrating that commencement of an interdisciplinary group intervention for patients with low levels of responsiveness is feasible in an acute hospital ward, this investigation has generated further empirical evidence highlighting the rehabilitation potential for patients who have suffered a severe brain injury (Groswasser \& Sazbon, 1990; Katz et al., 2009; Sazbon \& Groswasser, 1990). The long-term benefits of early and subacute rehabilitation that have been reported previously include improved functional independence within the home and potential return to work with a moderate quality of life (Groswasser \& Sazbon, 1990; Katz et al., 2009). The interdisciplinary intervention investigated in this study offers a potentially efficient and safe approach for augmenting the commencement of early rehabilitation, fostering family engagement in therapies, and fulfilling the National Health and Medical Research Council recommendations for the clinical management of this patient group in an acute hospital setting (National Health and Medical Research Council, 2008; Turner-Stokes et al., 2005).

This investigation has several strengths and limitations that influence the potential to generalise findings from this research. The small sample size, non-blinded outcome assessors and nonrandomised nature of the historical control comparator have been acknowledged as limitations that must be considered when interpreting findings from this research. Additionally, historical controls were only matched on GCS score. Other factors which may have influenced the potential for neurological recovery may include the type and nature of the brain injury (Husson et al., 2010). On the other hand, implementing a prospective design in a real-life neurosciences hospital unit and utilising existing clinical staff to provide the interdisciplinary intervention group within their usual caseload supports the feasibility of delivering this intervention in other similar clinical environments. Another key consideration is that the setting for this investigation was a tertiary facility in a first 
world country, and that therapists working in this unit have clinical experience working with this patient group in addition to their formal tertiary professional qualifications. The outcomes from this intervention may have not been the same in dissimilar settings or utilising dissimilar therapists. A further limitation, beyond the scope of this preliminary study, is the possibility that due to the increased focus on patients with severely altered consciousness and the implementation of the interdisciplinary group, other patients in the acute neurosciences ward may have received reduced therapy time.

Following this investigation there are several priorities for future research among patients with low levels of responsiveness following acute brain injury $(\mathrm{ABI})$. A larger randomised clinical trial evaluating the effectiveness of an interdisciplinary intervention among patients with low levels of responsiveness is warranted. Ideally, a large trial of this nature would involve multiple sites to ensure findings could be generalised widely. An additional consideration is the evaluation of rehabilitation intensity. This investigation observed an increase in intervention frequency delivered to patients in the prospective group. It is likely that increased therapy intensity may contribute to accelerated or enhanced recovery, but diminishing additional returns may begin to occur as the intensity of intervention delivery (frequency or duration of sessions) is increased. In the context of contemporary societies where demand for healthcare resources outweighs their availability, economic evaluation of early rehabilitation interventions for people with ABI is needed. Such evaluations would benefit from consideration of potential long-term costs averted in addition to the short-term costs of service provision. Long-term costs may include potential cost savings from reduced institutional care demands or productivity associated with lower care demands placed on family members of patients who received rehabilitation interventions. Future investigations among this group should also consider evaluation that extends beyond clinical outcome measures to include the perspective of the patient and family where possible. The inclusion of health-related quality of life evaluation would offer a useful mechanism to conduct economic analyses that can incorporate the lived experience of people with ABI through cost-utility analyses (McPhail, Beller, \& Haines, 2008).

In conclusion, this investigation has successfully demonstrated that implementing an interdisciplinary intervention in an acute neuroscience hospital ward within existing clinical human resourcing and without adverse events is feasible. Patients receiving this interdisciplinary interven- tion demonstrated substantial improvement in a wide range of clinical outcomes during the investigation, and a high proportion were subsequently discharged to subacute brain injury specific rehabilitation units. However, there is need for further research among this clinical group to investigate the effectiveness (including cost-effectiveness) and optimal intensity of early interdisciplinary rehabilitation for patients with low levels of responsiveness following $\mathrm{ABI}$.

\section{Acknowledgements}

This research was supported by the Queensland Department of Health (Health Practitioner Research Grant Scheme) and Queensland Neurosurgery \& Spine Surgery Foundation. S.M. is supported by a National Health and Medical Research Council Fellowship. The authors would also like to thank the staff of the Neurosciences Unit, Princess Alexandra Hospital for their contribution to this study.

\section{References}

Adair, J.G. (1984). The Hawthorne Effect: A reconsideration of the methodological artifact. Journal of Applied Psychology, 69(2), 334.

Cowen, T.D., Meythaler, J.M., DeVivo, M.J., Ivie, C.S., 3rd, Lebow, J., \& Novack, T.A. (1995). Influence of early variables in traumatic brain injury on functional independence measure scores and rehabilitation length of stay and charges. Archives of Physical Medicine and Rehabilitation, 76(9), 797-803.

Das-Gupta, R., \& Turner-Stokes, L. (2002). Traumatic brain injury. Disability and Rehabilitation, 24(13), 654-665.

DeFina, P.A., Fellus, J., Thompson, J.W., Eller, M., Moser, R.S., Frisina, P.G., .. . Prestigiacomo, C.J. (2010). Improving outcomes of severe disorders of consciousness. Restorative Neurology and Neuroscience, 28(6), 769-780.

Eilander, H.J., Timmerman, R.B., Scheirs, J.G., Van Heugten, C.M., De Kort, P.L., \& Prevo, A.J. (2007). Children and young adults in a prolonged unconscious state after severe brain injury: Long-term functional outcome as measured by the DRS and the GOSE after early intensive neurorehabilitation. Brain Injury, 21(1), 53-61.

Gesch, J., Nascimento, P., Passier, L., \& Haines, T. (2005). Acute brain injury physiotherapy assessment: A new scale for an old problem [2005 National Neurology \& Gerontology Joint Conference Abstracts]. Australian Journal of Physiotherapy, 51(eSupplement).

Giacino, J.T., Ashwal, S., Childs, N., Cranford, R., Jennett, B., Katz, D.I., ... Zasler, N.D. (2002). The minimally conscious state: Definition and diagnostic criteria. Neurology, 58, 349-353. 
Gill, M.R., Reiley, D.G., \& Green, S.M. (2004). Interrater reliability of Glasgow Coma Scale scores in the Emergency Department. Annals of Emergency Medicine, 43, 215-223.

Groswasser, Z., \& Sazbon, L. (1990). Outcome in 134 patients with prolonged posttraumatic unawareness. Part 2: Functional outcome of 72 patients recovering consciousness. Journal of Neurosurgery, 72(1), 8184.

Husson, E.C., Ribbers, G.M., Willemse-van Son, A.H., Verhagen, A.P., \& Stam, H.J. (2010). Prognosis of six-month functioning after moderate to severe traumatic brain injury: A systematic review of prospective cohort studies. Journal of Rehabilitation Medicine, 42(5), 425-436.

Kalmar, K., \& Giacino, J.T. (2005). The Coma Recovery Scale - Revised. Neuropsychology Rehabilitation, 15(3-4), 454-460.

Katz, D.I., Polyak, M., Coughlan, D., Nichols, M., \& Roche, A. (2009). Natural history of recovery from brain injury after prolonged disorders of consciousness: Outcome of patients admitted to inpatient rehabilitation with 1-4 year follow-up. Progress in Brain Research, 177, 73-88.

Kidd, D., Stewart, G., Baldry, J., Johnson, J., Rossiter, D., Petruckevitch, A., \& Thompson, A. (1995). The Functional Independence Measure: A comparative validity and reliability study. Disability and Rehabilitation, 17(1), 454-460.

Lippert-Gruner, M., Wedekind, C., \& Klug, N. (2002). Functional and psychosocial outcome one year after severe traumatic brain injury and early-onset rehabilitation therapy. Journal of Rehabilitation Medicine, 34(5), 211-214.

Low Choy, N., Kuys, S., Richards, M., \& Isles, R. (2002). Measurement of functional ability following traumatic brain injury using the Clinical Outcomes Variable Scale: A reliability study. Australian Journal of Physiotherapy, 48, 35-39.

Mackay, L.E., Bernstein, B.A., Chapman, P.E., Morgan, A.S., \& Milazzo, L.S. (1992). Early intervention in severe head injury: long-term benefits of a formalized program. Archives of Physical Medicine and Rehabilitation, 73, 635-641.

Mayo, E. (1933). The human problems of an industrial civilization. New York: McMillan.

McPhail, S., Beller, E., \& Haines, T. (2008). Two perspectives of proxy reporting of health-related quality of life using the Euroqol-5D, an investigation of agreement. Medical Care, 46(11), 1140-1148.

Meyer, M.J., Megyesi, J., Meythaler, J.M., MurieFernandez, M., Aubut, J., Foley, N., ... Teasell, R. (2010). Acute management of acquired brain injury
Part III: An evidence-based review of interventions used to promote arousal from coma. Brain Injury, 24(5), 722-729.

National Health and Medical Research Council. (2008). Ethical guidelines for the care of people in post-coma unresponsiveness (vegetative state) or a minimally responsive state. Canberra: Australian Government Publishing Service.

Prasad, K. (1996). The Glasgow Coma Scale: A critical appraisal of its clinimetric properties. Journal of Clinical Epidemiology, 49(7), 755-763.

Reid-Arndt, S.A., Schopp, L., Brenneke, L., Johnstone, B., \& Poole, D. (2007). Evaluation of the Traumatic Brain Injury Early Referral Program in Missouri. Brain Injury, 21(12), 1295-1302.

Sazbon, L., \& Groswasser, Z. (1990). Outcome in 134 patients with prolonged posttraumatic unawareness. Part 1: Parameters determining late recovery of consciousness. Journal of Neurosurgery, 72(1), 7580.

Seaby, L., \& Torrance, G. (1989). Reliability of a physiotherapy functional assessment used in a rehabilitation setting. Physiotherapy Canada, 41, 264271.

Shiel, A., Burn, J.P., Henry, D., Clark, J., Wilson, B.A., Burnett, M.E., \& McLellan, D.L. (2001). The effects of increased rehabilitation therapy after brain injury: Results of a prospective controlled trial. Clinical Rehabilitation, 15(5), 501-514.

Sorbo, A., Rydenhag, B., Sunnerhagen, K.S., Blomqvist, M., Svensson, S., \& Emanuelson, I. (2005). Outcome after severe brain damage, what makes the difference? Brain Injury, 19(7), 493-503.

Teasdale, G., \& Jennett, B. (1974). Assessment of coma and impaired consciousness. A practical scale. Lancet, 2, 81-84.

Turner-Stokes, L. (2008). Evidence for the effectiveness of multi-disciplinary rehabilitation following acquired brain injury: A synthesis of two systematic approaches. Journal of Rehabilitation Medicine, 40(9), 691-701.

Turner-Stokes, L., Disler, P B., Nair, A., \& Wade, D.T. (2005). Multi-disciplinary rehabilitation for acquired brain injury in adults of working age. Cochrane Database of Systematic Reviews, 20(3).

Ward, E.C., \& Conroy, A.-L. (1999). Validity, reliability and responsivity of the Royal Brisbane Hospital Outcome Measure for Swallowing. Asia Pacific Journal of Speech, Language and Hearing, 4, 109-129.

Wilson, L.A., \& Brass, W. (1973). Brief assessment of the mental status in geriatric domicilary practice. The usefulness of the Mental Status Questionnaire. Age and Ageing, 2, 92-101. 